\title{
Linear criteria for gravity-wave breaking in resonant stratified flow over a ridge
}

Article

Accepted Version

Teixeira, M. A. C. and Miranda, P. M. A. (2005) Linear criteria for gravity-wave breaking in resonant stratified flow over a ridge. Quarterly Journal of the Royal Meteorological Society, 131 (609). pp. 1815-1820. ISSN 1477-870X doi:

https://doi.org/10.1256/qj.04.155 Available at https://centaur.reading.ac.uk/29250/

It is advisable to refer to the publisher's version if you intend to cite from the work. See Guidance on citing.

To link to this article DOI: http://dx.doi.org/10.1256/qj.04.155

Publisher: Royal Meteorological Society

All outputs in CentAUR are protected by Intellectual Property Rights law, including copyright law. Copyright and IPR is retained by the creators or other copyright holders. Terms and conditions for use of this material are defined in the End User Agreement.

\section{www.reading.ac.uk/centaur}

\section{CentAUR}

Central Archive at the University of Reading 
Reading's research outputs online 


\section{Linear criteria for gravity wave breaking in resonant stratified flow over a ridge}

By M. A. C. Teixeira* and P. M. A. Miranda

Geophysics Centre and Department of Physics, University of Lisbon, Portugal

(Received 1 January 2002; revised 1 January 2002)

\section{SUMMARY}

Using linear theory, it is shown that, in resonant flow over a $2 \mathrm{D}$ mountain ridge, such as exists when a layer of uniform wind is topped by an environmental critical level, the conditions for internal gravity wave breaking are different from those determined in previous studies for non-resonant flows. For Richardson numbers in the shear layer not exceeding 2.25 , two zones of flow overturning exist, respectively below and downstream and above and upstream of the expected locations. Flow overturning occurs for values of the dimensionless height of the ridge smaller than those required for a uniform wind profile. These results may have implications for the physical understanding of high-drag states.

KEYwords: Mountain waves, Flow stagnation, Critical level

\section{INTRODUCTION}

Knowing under what conditions topographically generated gravity waves overturn is of great practical importance. This is because, in principle, no steady waves can exist when these conditions are met, since zones of static instability are generated, which lead to wave breaking. Additionally, flow overturning generates critical levels, which are thought to play a crucial role in nonlinear high-drag states. Clark and Peltier (1984) suggested that these self-induced critical levels reflect gravity waves downward, thereby allowing a reinforcement of the drag signal. In hydrostatic, constant-velocity flow over a $2 \mathrm{D}$ bell-shaped ridge, linear theory predicts that gravity waves overturn exactly above the ridge top, at a height of 0.75 times the vertical wavelength, $\lambda=2 \pi U_{0} / N$, and for dimensionless mountain heights larger than $N h_{0} / U_{0}=1$ (where $N$ and $U_{0}$ are the ambient Brunt-Väisälä frequency and windspeed, and $h_{0}$ is the height of the ridge top). Nonlinear theory predicts instead that flow overturning first occurs slightly downstream of the ridge top, at $x / a=0.33$ (where $a$ is the ridge half-width), at a height $z / \lambda=0.76$ (Laprise and Peltier 1989) and for $N h_{0} / U_{0}>0.85$ (Miles and Huppert 1969). These are considered reference values, and have been used, namely, to physically interpret the behaviour of the drag in flows with environmental critical levels (Bacmeister and Pierrehumbert 1988, Scinocca and Peltier 1991). However, it will be shown in this note that, since such flows support resonance, the zones where wave overturning first occurs, and the corresponding critical $N h_{0} / U_{0}$, are not fixed, and may be different from those quoted above. This may help to explain the locations of drag maxima in previous studies addressing the problem of high-drag states.

\section{THE MODEL}

A linear, hydrostatic, Boussinesq, 2D model similar to that employed by Wang and Lin (1999a) and Teixeira et al. (2005) is adopted here (the hydrostatic approximation is valid provided that vertical accelerations are not too large). The flow perturbations, which are constant in the $y$ direction, are Fourier analysed along $x$, and their Fourier transforms are denoted by a hat. The vertical velocity perturbation, $\hat{w}$, satisfies the hydrostatic Taylor-Goldstein equation,

$$
\hat{w}^{\prime \prime}+\frac{N^{2}}{U^{2}}-\frac{U^{\prime \prime}}{U} \quad \hat{w}=0,
$$

where the primes denote differentiation with respect to $z$ and $U$ is the background wind (assumed to be along $x$ ).

Following Teixeira et al. (2005), the simplest background wind profile capable of generating wave resonance is considered:

$$
U= \begin{cases}U_{0} & \text { if } \quad z \leqslant z_{1} \\ U_{0} & \frac{z_{c}-z}{z_{c}-z_{1}} \quad \text { if } \quad z>z_{1} .\end{cases}
$$

* Corresponding author: Centro de Geofísica da Universidade de Lisboa, Edifício C8, Campo Grande, 1749-016 Lisbon, Portugal.

(c) Royal Meteorological Society, 2002. 
The wind velocity is constant near the surface, and above $z=z_{1}$ decreases linearly. As shown by Teixeira et al. (2005), when $R i \geqslant 0.5$, this wind profile is approximately equivalent, concerning the behaviour of the waves in the lower layer, to a profile that differs from it above the critical level, $z_{c}$, because the waves are absorbed almost perfectly at $z_{c}$. This wind profile may not be realistic for relatively low Richardson numbers, $R i=N^{2}\left(z_{c}-z_{1}\right)^{2} / U_{0}^{2}$, since then the critical level becomes much more transparent and wave reflections occurring above it may matter.

For the flow (2), the solution of $(1)$ in the lower layer $\left(z<z_{1}\right)$ is

$$
\hat{w}=\alpha(k) e^{i \frac{N}{U_{0}} \operatorname{sgn}(k) z}+\beta(k) e^{-i \frac{N}{U_{0}} \operatorname{sgn}(k) z},
$$

while in the upper layer $\left(z>z_{1}\right)$ it is

$$
\hat{w}=\gamma(k) \frac{z}{c}-z_{z_{c}-z_{1}}^{1 / 2-i \mu \operatorname{sgn}(k)}
$$

where $\mu=(R i-1 / 4)^{1 / 2}$. In these solutions, $k$ is the horizontal wavenumber, $\alpha, \beta$ and $\gamma$ are functions to be determined by the boundary conditions, and the radiation boundary condition in the upper layer is implicitly assumed. The remaining boundary conditions are that both $\hat{w}$ and $\hat{p}$ (the Fourier transform of the pressure perturbation) are continuous at $z=z_{1}$ and that the flow follows the topography at the surface:

$$
\hat{w}(z=0)=i U_{0} k \hat{\eta},
$$

where $\hat{\eta}$ is the Fourier transform of the terrain elevation. When these boundary conditions are applied, the following equations for $\alpha, \beta$ and $\gamma$ are obtained:

$$
\begin{aligned}
& \alpha=\frac{U_{0} k \hat{\eta}\left[i N+\frac{U_{0}}{z_{c}-z_{1}} \frac{1}{2}+i \mu\right.}{\operatorname{sgn}(k)] e^{-i \frac{N}{U_{0}} \operatorname{sgn}(k) z_{1}}},
\end{aligned}
$$

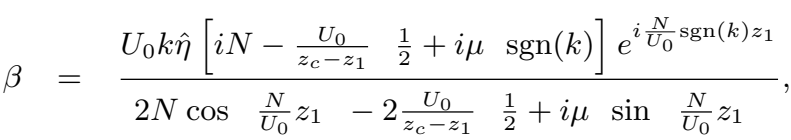

$$
\begin{aligned}
& \gamma=\frac{i U_{0} k \hat{\eta} N}{N \cos \frac{N}{U_{0}} z_{1}-\frac{U_{0}}{z_{c}-z_{1}} \frac{1}{2}+i \mu \sin \frac{N}{U_{0}} z_{1}} .
\end{aligned}
$$

This totally specifies the solution to the internal gravity wave problem.

Since in this study the focus is on flow overturning, the streamwise velocity perturbation, $u$, is calculated, because it is this variable that determines flow stagnation. The corresponding Fourier transform can be related to $\hat{w}$ by

$$
\hat{u}=i \frac{\hat{w}^{\prime}}{k},
$$

from mass conservation. For a bell shaped ridge, the Fourier transform of the terrain elevation has the form

$$
\hat{\eta}=\frac{h_{0} a}{2} \mathrm{e}^{-a|k|} .
$$

For this particular case, the streamwise velocity perturbation can be determined analytically from (3), (6)-(7), (9) and (10) and taking the inverse Fourier transform, yielding, in the lower layer:

$$
\begin{aligned}
u= & \frac{N h_{0}}{\left[1+\frac{x}{a}^{2}\right]\left[1-\frac{1}{2} R i^{-1 / 2} \sin 2 \frac{N z_{1}}{U_{0}}\right]} \quad 1-\frac{1}{2} R i^{-1 / 2} \sin 2 \frac{N z_{1}}{U_{0}} \sin \frac{N z}{U_{0}} \\
& +\mu \frac{x}{a}-\frac{1}{2} \cos 2 \frac{N z_{1}}{U_{0}} \quad R i^{-1 / 2} \cos \frac{N z}{U_{0}} .
\end{aligned}
$$

In the upper layer, flow overturning always occurs, because the waves steepen indefinitely before they reach the critical level. However, this steepening seems not to have a very important impact on the surface drag in some situations, as shown by the fact that linear theory can predict the drag well for sufficiently low mountains (Teixeira et al. 2005). So attention will be focused only on flow overturning in the lower, constant-wind layer. 


\section{FLOW OVERTURNING CONDITION}

The critical conditions for flow overturning are:

$$
\frac{\partial u}{\partial x}=0, \quad \frac{\partial u}{\partial z}=0, \quad u=-U_{0} .
$$

The first two conditions ensure that an extremum (or saddle point) of $u$ is selected. This includes the $u$ minima where flow stagnation first occurs. The third condition ensures that this situation corresponds to vertical streamlines. While the use of linear theory is questionable in such extreme conditions, this approach has proved its value in analogous problems (Smith 1989). Of course, the predictions resulting from this model can only be expected to have a qualitative value.

From the first two conditions of (12), the horizontal positions of the maximum horizontal flow perturbations are given by the solution of

$$
\begin{array}{ll}
1-\frac{1}{4} R i^{-1} \quad \frac{x}{a}-\frac{3}{2} R i^{-1} \mu \cos \quad 2 \frac{N z_{1}}{U_{0}} \quad \frac{x}{a}^{2} \\
+1+\frac{3}{4} R i^{-1}-2 R i^{-1 / 2} \sin \quad 2 \frac{N z_{1}}{U_{0}} \quad \frac{x}{a}+\frac{1}{2} R i^{-1} \mu \cos \quad 2 \frac{N z_{1}}{U_{0}}=0 .
\end{array}
$$

If this solution is denoted by $x_{s}$, then the corresponding vertical locations, $z_{s}$, are given by the solution of

$$
\tan \frac{N z_{s}}{U_{0}}=\frac{1-\frac{1}{2} R i^{-1 / 2} \sin 2 \frac{N z_{1}}{U_{0}}}{\left[\mu \frac{x_{s}}{a}-\frac{1}{2} \cos 2 \frac{N z_{1}}{U_{0}}\right] R i^{-1 / 2}} .
$$

Additionally, the third condition implies that the critical dimensionless mountain height is given by

$$
\frac{N h_{0}}{U_{0}}=-\frac{\left[1+{\frac{x_{s}}{a}}^{2}\right]\left[1-\frac{1}{2} R i^{-1 / 2} \sin 2 \frac{N z_{1}}{U_{0}}\right]}{\left[1-\frac{1}{2} R i^{-1 / 2} \sin 2 \frac{N z_{1}}{U_{0}}\right] \sin \frac{N z_{s}}{U_{0}}+\left[\mu \frac{x_{s}}{a}-\frac{1}{2} \cos 2 \frac{N z_{1}}{U_{0}}\right] R i^{-1 / 2} \cos \frac{N z_{s}}{U_{0}}} .
$$

From (13), (14) and (15), it can be seen that both the location of the zones of critical steepness and the critical $N h_{0} / U_{0}$ depend on $R i$ and on $N z_{1} / U_{0}$ (as happened with the surface drag in the study of Teixeira et al. 2005). Since (13) is a cubic equation, its solution is in general too complicated to be useful for practical purposes. However, it is known from Teixeira et al. (2005) that the flow perturbations become more intense as one approaches the drag maxima, which are located at $N z_{1} / \pi U_{0}=0.25+n$, where $n$ is an integer. For the drag minima, $N z_{1} / \pi U_{0}=0.75+n$. In both of these cases, one of the roots of (13) becomes $x / a=0$, and the remaining two roots are given by a second order equation, which is much more manageable. Since these cases can be considered as upper and lower limits for the likeliness of overturning, attention will be focused on them henceforth.

At the drag minima, the situation most unfavourable for flow overturning, (13) only has one real root: $x_{s} / a=0$. Along with (14) and (15), this leads to $N z_{s} / \pi U_{0}=1.5+2 n$ and $\left(N h_{0} / U_{0}\right)_{s}=1$. This is the usual condition for critical flow in linear theory.

At the drag maxima, two possibilities exist. For $R i>9 / 4$, the only real root of (13) is once again $x_{s} / a=0$, which also leads to $N z_{s} / \pi U_{0}=1.5+2 n$ and $\left(N h_{0} / U_{0}\right)_{s}=1$. However, when $1 / 4<R i<9 / 4$, there are three possible real roots to (13). $x_{s} / a=0$ (which now corresponds to a saddle point of the $u$ field) and

$$
\left.\frac{x_{s}}{a}=-\frac{\frac{3}{2}-R i^{1 / 2}}{R i^{1 / 2}+\frac{1}{2}}\right)^{1 / 2} \quad \text { or } \quad \frac{x_{s}}{a}=+\left(\frac{\frac{3}{2}-R i^{1 / 2}}{R i^{1 / 2}+\frac{1}{2}}\right)^{1 / 2}
$$

which correspond to maxima and minima. For the $x_{s} / a$ given by (16), the $N z_{s} / \pi U_{0}$ pertaining to the $u$ minima are given, in the same order, as

$$
\begin{aligned}
& \left.\left.\frac{N z_{s}}{\pi U_{0}}=-\frac{1}{\pi} \arctan \left[\frac{R i^{1 / 2}-\frac{1}{2}}{\frac{3}{2}-R i^{1 / 2}}\right)^{1 / 2}\right]+2 n \quad \text { or } \quad \frac{N z_{s}}{\pi U_{0}}=+\frac{1}{\pi} \arctan \left[\frac{R i^{1 / 2}-\frac{1}{2}}{\frac{3}{2}-R i^{1 / 2}}\right)^{1 / 2}\right] \\
& +(2 n+1) \text {, }
\end{aligned}
$$




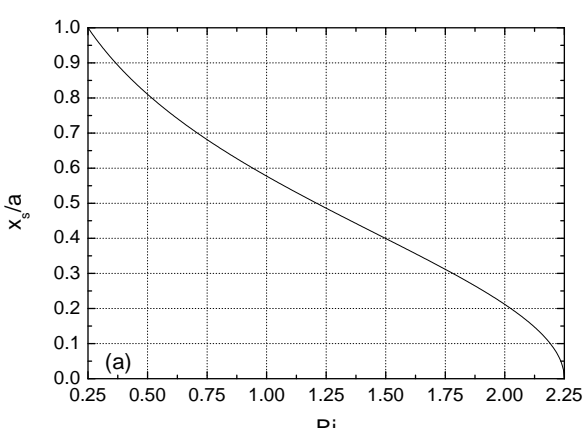

$\mathrm{Ri}$

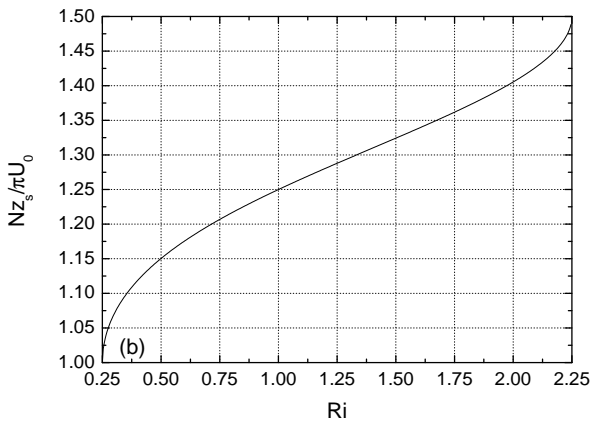

$\mathrm{Ri}$

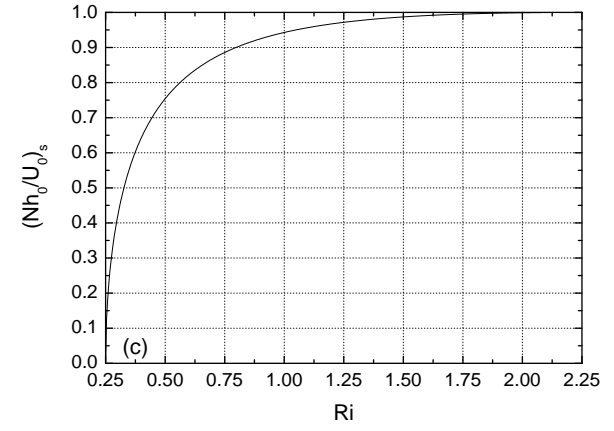

Figure 1. Characteristics of the lowest flow overturning zone for the drag maxima $\left(N z_{1} / \pi U_{0}=0.25+\right.$ $n$ ), as a function of $R i$. (a) normalised $x$ position, (b) normalised $z$ position, (c) critical dimensionless mountain height.

where $n$ is an integer. The corresponding critical mountain height is

$$
\frac{N h_{0}}{U_{0}}{ }_{s}=2 \frac{R i^{1 / 2}-\frac{1}{2}^{1 / 2}}{R i^{1 / 2}+\frac{1}{2}} .
$$

These expressions show that, when $R i$ drops below $9 / 4$, the zones of flow overturning are displaced symmetrically upward and upstream and downward and downstream relative to their expected location. This displacement becomes more pronounced as $R i$ decreases. The critical $N h_{0} / U_{0}$ is also reduced. For example, when $R i=1 / 4$ (a rather extreme case), the flow overturns at $x_{s} / a= \pm 1$ and $N z_{s} / \pi U_{0}=n$, with alternate signs of $x_{s} / a$ (beginning with positive) corresponding to consecutive values of $n$ (for $n \geqslant 1$ ).

Figure 1 shows the location and critical mountain height of the first (lowest) wave overturning level, between $R i=1 / 4$ and $R i=9 / 4$. It can be seen in Fig. 1(a) that $x_{s} / a$ goes smoothly from $x_{s} / a=0$, at $R i=9 / 4$ to $x_{s} / a=1$, for $R i=1 / 4$. In the same parameter range, $N z_{s} / \pi U_{0}$ decreases from 1.5 to 1 (Fig. 1(b)), and $\left(N h_{0} / U_{0}\right)_{s}$ decreases from 1 to 0 (Fig $1(\mathrm{c})$ ). The first two results are a manifestation of the interference of upward and downward propagating waves in the constant-wind layer. The physical interpretation of the last result is the following. Teixeira et al. (2005) have shown that, for the type of flow assumed here and for $R i=1 / 4$, the drag maxima tend to infinity, due to total wave reflection at $z=z_{1}$. Therefore, the associated flow perturbations (including $u$ ) also tend to infinity. Then, no matter how small $N h_{0} / U_{0}$ is, there will always be flow overturning.

When $R i=1$, such as happens in various studies about high-drag states with environmental critical levels, $x_{s} / a \approx 0.57, N z_{s} / \pi U_{0} \approx 1.25$ and $\left(N h_{0} / U_{0}\right)_{s}=0.94$. When $R i=0.5$, on the other hand, a situation where the critical level is still approximately an absorber, $x_{s} / a=0.81$, $N z_{s} / \pi U_{0}=1.15$ and $\left(N h_{0} / U_{0}\right)_{s}=0.75$.

Figure 2 illustrates the flow configuration for $R i=0.5$, which differs markedly from the configuration for non-resonant situations. The isentropes (lines of constant potential temperature) are displayed for $N z_{1} / \pi U_{0}=3.25$, corresponding to a drag maximum, for 3 different values of $N h_{0} / U_{0}$. The isentropes coincide with streamlines, since the flow is adiabatic. In Fig. 

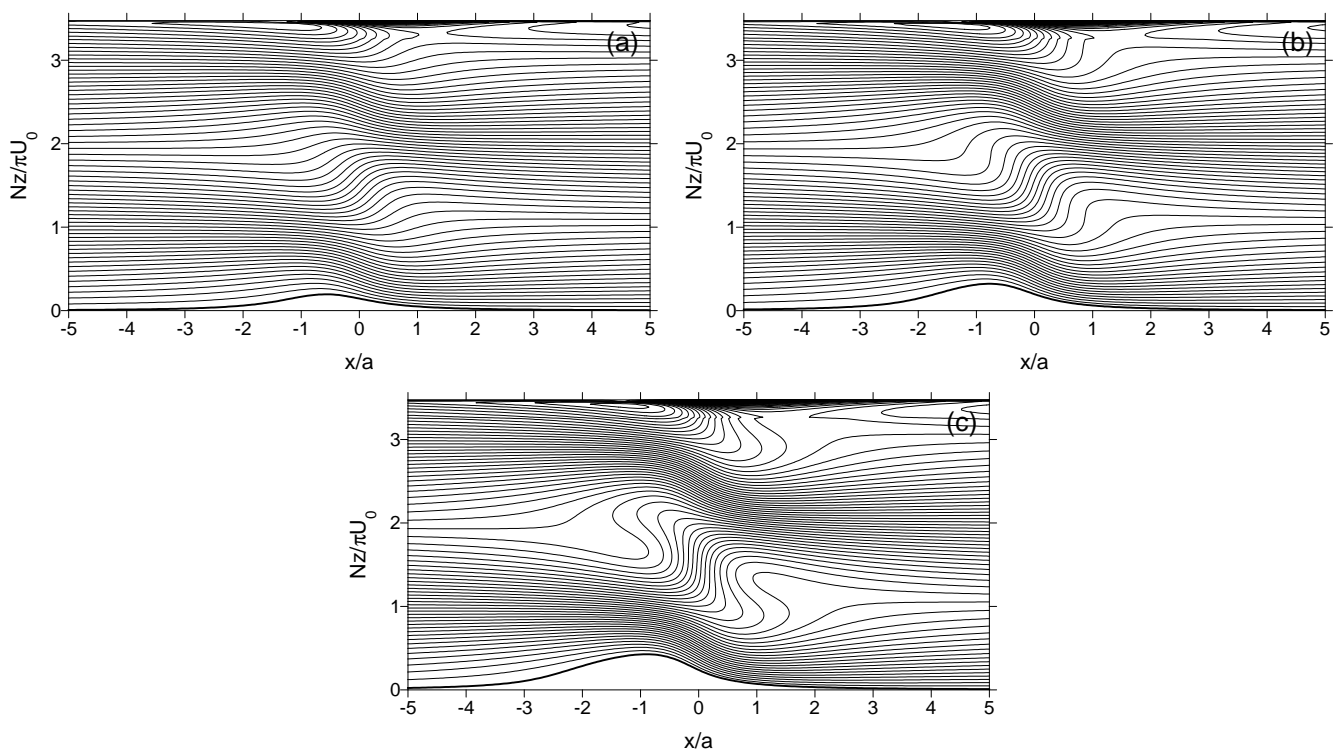

Figure 2. Isentropes, with a spacing of $0.5 \mathrm{~K}$, for flow with $R i=0.5$ and $N z_{1} / \pi U_{0}=3.25$. (a) $N h_{0} / U_{0}=$ 0.5 , (b) $N h_{0} / U_{0}=0.75$, (c) $N h_{0} / U_{0}=1$. The surface streamline does not follow the terrain elevation (centred on $x / a=0$ ) because of the linearised boundary condition.

$2(\mathrm{a})$, it can be seen that, for $N h_{0} / U_{0}=0.5$, when the overturning condition is not satisfied, the minima of $u$ (that correspond to the zones of larger streamline spacing) have a streamline slope lower than that existing at $x / a=0$ and $N z / \pi U_{0}=1.5$. However, when $N h_{0} / U_{0}=0.75$ (close to the overturning condition) (Fig. 2(b)), the zones of maximum streamline slope are located as predicted by (16)-(17). In these regions the streamline slope is approximately infinite, while in between, in the traditionally expected wave breaking location, it is still finite. Finally, in Fig. 2(c), for $N h_{0} / U_{0}=1$, the streamlines have overturned at the points defined by (16)-(17), but are vertical in the middle, in the zone of traditionally expected wave breaking. This last panel illustrates clearly how wave breaking first occurs indeed in zones displaced laterally from the ridge top. A curious detail, which may be checked using (15) is that, for $N h_{0} / U_{0}=1$, the streamline slope at $x / a=0$ and $N z / \pi U_{0}=1.5$ is always infinite, as in non-resonant situations.

\section{DisCUSSION}

Most studies addressing the problem of high-drag states use environmental critical levels to induce these states, and much of their physical interpretation is based on the notion that wave overturning occurs, in a constant-wind layer, at the level $N z_{s} / \pi U_{0}=1.5$. The present study has shown that this is not necessarily so. In resonant flow, the zones of critical streamline slope are displaced both horizontally and vertically relative to a non-resonant flow, as long as $R i<9 / 4$. This is due to the two-lobe structure of the $u$ velocity perturbation that exists when there is sufficient constructive interference between the upward and downward propagating waves in the region $z<z_{1}$ (cf. Fig. 3 of Teixeira et al. 2005). Obviously, this finding has great relevance for the interpretation of high-drag states. If it is accepted that the self-induced critical levels determine the locations of the drag maxima, the present results suggest that these locations should depend on Ri. In particular the migration of the drag maxima to the left in Fig. 2 of Bacmeister and Pierrehumbert (1988) could be partly attributed to the decrease of the Richardson number from 2.25 to 1 between those two curves. Admittedly, there is conflicting evidence, as proved by the fact that the first drag maximum shifts its location only slightly to the left (when scaled by $z_{c}$ ) as $R i$ decreases in Fig. 5(b) of Teixeira et al. (2005).

Additionally, the present model indicates that the critical value of $N h_{0} / U_{0}$ for wave breaking decreases as $R i$ decreases. This appears to be consistent with Fig. 2 of Wang and Lin (1999b), where it can be seen that high-drag states become more likely for low $R i$. The 
usefulness of Fig. 1(c) of this study could perhaps be extended if instead of interpreted literally as the critical dimensionless mountain height, the quantity on the vertical axis is interpreted as the critical mountain height divided by its value in non-resonant flow. Knowing that from the nonlinear theory of Miles and Huppert (1969) $\left(N h_{0} / U_{0}\right)_{s}=0.85$ when there is no resonance, this would enable one to extrapolate that, for $R i=1,\left(N h_{0} / U_{0}\right)_{s} \approx 0.8$ and for $R i=0.5$, $\left(N h_{0} / U_{0}\right)_{s} \approx 0.64$ in resonant flow. These inferences may not be of direct help in analysing strongly nonlinear flows, but they may give hints as to what linear processes trigger the observed nonlinear behaviour.

\section{ACKNOWLEDGEMENTS}

This work was supported by Fundação para a Ciência e Tecnologia (FCT) under project BULET/33980/99, co-financed by the European Union under program FEDER. MACT acknowledges the financial support of FCT under grant SFRH/BPD/3533/2000.

Bacmeister, J. T. and

Pierrehumbert, R. T.

Clark, T. L. and Peltier, W. R.

Laprise, R. and Peltier, W. R.

Miles, J. W. and Huppert, H. E. 1969

Scinocca, J. F. and Peltier, W. 1991 R.

Smith, R. B.

Teixeira, M. A. C., Miranda, P. M. A., Argain, J. L. and Valente, M. A.

Wang, T.-A. and Lin, Y.-L.

Wang, T.-A. and Lin, Y.-L.

1988

1984

1989

1989

2005

\section{REFERENCES}

1999a Atmos, Sci., 56, 412-436

1999b Wave ducting in a stratified shear flow over a twodimensional mountain. Part II: Implications for the development of high-drag states for severe downslope windstorms. J. Atmos. Sci., 56, 437-452 